\title{
On soluble groups which admit the dihedral group of order eight fixed-point-freely
}

\section{Alan R. Camina and F. Peter Lockett}

\begin{abstract}
If the finite soluble group $G$ admits the dihedral group of order eight as a fixed-point-free group of automorphisms then the nilpotent length of $G$ is at most three.
\end{abstract}

A theorem of Berger [2] has substantially enlarged the class of nilpotent groups $A$ for which the following statement holds.

(*) If the soluble group $G$ admits the group $A$ as a fixed-pointfree group of automorphisms and $(|G|,|A|)=1$, then the nilpotent length of $G$ is bounded by the number of primes, including multiplicities, which divide $|A|$.

The smallest group $A$ not covered by Berger's result is $D_{8}$, the dihedral group of order 8 . It is our object here to establish (*) when $A=D_{8}$. In [5] Gross shows that, in this case, 4 is a bound, and in this paper he provides an important step in our argument.

$F_{1}(G), F_{2}(G), \ldots$ (or often just $F_{1}, F_{2}, \ldots$ when no confusion arises) will denote the successive terms of the upper nilpotent series of the soluble group $G$, and f.p.f. will be used to abbreviate both "fixedpoint-free" and "fixed-point-freely". $\Phi(H)$ will denote the Frattini subgroup of $H$. All groups considered will be finite.

THEOREM. If the soluble group $G$ admits $D_{8}$ as a fixed-point-free 
group of automorphisms then the nilpotent length of $G$ is at most 3 .

Proof. Let $G$ be a minimal counterexample to the theorem, so $G$ has nilpotent length 4 and each $D_{8}$-admissible proper section of $G$ has nilpotent length 3 . Let $D_{8}=\left\langle\tau, n: \tau^{4}=1=\eta^{2}, \tau^{n}=\tau^{-1}\right)$ and put $\sigma=\tau^{2}$, the central involution. The hypothesis of f.p.f. action implies that $G$ has odd order.

We first apply Theorem 2.4, Corollary 2.5 and Lemma 2.6 of Gross [6] to achieve a major part of the reduction.

(1) $G=S R Q P$ where $S, R, Q$ and $P$ are $D_{8}$-admissible subgroups of $G$ and:

(a) $S$ is an s-group, $R$ is an r-group, $Q$ is a q-group and $P$ is a p-group;

(b) $s, r, q$ and $p$ are primes with $s \neq r \neq q \neq p$;

(c) $P$ normalizes $Q, R$ and $S ; Q$ normalizes $R$ and $S$; and $R$ normalizes $S$;

(d) $S \leq F_{1}(G), \quad R \leq F_{2}(G), \quad R \neq F_{1}(G), Q \leq F_{3}(G), Q \neq F_{2}(G)$, $P \neq F_{3}(G)$;

(e) $[Q, P]=Q,[R, Q]=R$ and $[S, R]=S$;

(f) each proper $D_{8}$-admissible subgroup of $P$ lies in $F_{3} ; P / P \cap F_{3}$ is elementary abelian and $D_{8}$-irreducible;

(g) for each proper $P D_{8}$-admissible subgroup $Q_{1}$ of $Q$, $\left[Q_{1}, P\right] \leq F_{2}$ and $Q / Q \cap F$ is a special q-group with $P D_{8}-$ irreducible Frattini quotient;

(h) for each proper $Q P D_{8}$-admissible subgroup $R_{1}$ of $R$, $\left[R_{1}, Q\right] \leq F_{1}$ and $R / R \cap F_{1}$ is a special r-group with $Q P D_{8}-$ irreducible Frattini quotient;

(i) $R$ centralizes each proper $R Q P D_{8}$-adnissible subgroup of $S$ and $S$ is a special s-group with $R Q P D_{8}$-irreducible Frattini 
quotient.

(2) $S=F_{1}(G)$ and is a faithful irreducible $R Q P D_{8}$-module.

If $\Phi(S) \neq 1, G / \Phi(S)$ has nilpotent length 3 by the minimality of $G$. Now $\Phi(S) \leq \Phi(G)$, so in this case $G / \Phi(G)$ has nilpotent length 3 , from which it follows that $G$ does too, a contradiction. Therefore by (li), $S$ is an elementary abelian 6 -group irreducible under the action of $R Q P D_{8}$. Clearly the minimality of $G$ implies that $G D_{8}$ has a unique minimal normal 2-subgroup, which must therefore be $S$. In particular $R \cap F_{1}=1$. Certainly $G D_{8}$ can have no normal 2-subgroup for otherwise $G$ would admit the four-group $D_{8} /(\sigma)$ f.p.f. contrary to a theorem of Bauman [1], which states that such groups have nilpotent derived group. It is now sufficient to prove that $R Q P$ complements $S$ in $G$ (for then $G D_{8}$ is a primitive soluble group with self-centralizing unique minimal normal subgroup $S$ ) and this will hold if $Q \cap S=I=P \cap S$. By (1c), $[Q \cap S, R] \leq R \cap S=1$ but $[S, R]=S$ by (le), so the irreducibility of $S$ forces $Q \cap S=1$. Similarly $[P \cap S, R] \leq R \cap S=1$ implies $P \cap S=1$.

(3) $\sigma$ centralizes $Q P$.

$P / \Phi(P)$ is a completely reducible $D_{8}$-module. If it were not $D_{8}-$ irreducible (lf) would force $P \leq F_{3}$, against (ld). So $P / \Phi(P)$ is $D_{8}-$ irreducible and therefore $P \cap F_{3} \leq \Phi(P)$. Since $p \neq q, Q / \Phi(Q)$ is a completely reducible $P D_{8}$-module. If it were not irreducible, say $Q / \Phi(Q)=Q_{1} / \Phi(Q)+Q_{2} / \Phi(Q)$ where $Q \neq Q_{1}, Q_{2}$, then (le) and (lf) would imply $Q=\left[Q_{1} Q_{2}, P\right]=\left[Q_{1}, P\right]\left[Q_{2}, P\right] \leq E_{2}$, against (1d). So $Q / \Phi(Q)$ is $P D_{8}$-irreducible and therefore $Q \cap F_{2} \leq \Phi(Q)$.

We may apply Theorem 1 of Gross [5] to the Group $R Q P D_{8}$, which by (2) acts faithfully and irreducibly on $S$, to deduce that $\sigma$ centralizes $E_{3} / F_{2}$. Since $G$ is a $2^{\prime}$-group it follows immediately that $\sigma$ centralizes $G / F_{2}$ and hence that $\sigma$ centralizes $P / P \cap F_{3}$ and $Q / Q \cap F_{2}$. But then, in view of the inclusions proved above, $\sigma$ centralizes $P / \Phi(P)$ and $Q / \Phi(Q)$ yielding the statement (3). 
(4) $R$ is a special group of exponent $r, \sigma$ inverts $R / \Phi(R)$ and centralizes $\Phi(R)$.

By (2), $R \cap F_{I}=1$, so (1h) says that $R$ is a special group whose Frattini quotient is isomorphic to a chief factor of $G D_{8}$. If $\Omega_{\perp}(R)=\left\langle x \in R: x^{2}=I\right\rangle$ were a proper subgroup of $R$ then $\left[\Omega_{1}(R), Q\right]=1$ by $(I h)$, therefore by 5.3 .10 of [4], Q would centralize $R$, contrary to (le). Thus $R$ is a special group generated by elements of order $r$, so it has exponent $r$. $\sigma$ does not centralize $R / \Phi(R)$, otherwise the group $R Q P$ of nilpotent length 3 admits the four-group $D_{8} /\langle\sigma\rangle$ f.p.f. again contrary to Bauman's Theorem. Now by (3), $\sigma$ is central in $Q P D_{8}$ so $C_{R / \Phi(R)}(\sigma)$ is normalized by $Q P D_{8}$, so by the irreducibility of $R / \Phi(R)$ this group is trivial. Thus $\sigma$ inverts each element of $R / \Phi(R) . R$ has class 2 so if $x, y \in R$ then $[x, y]^{\sigma}=\left[x^{\sigma}, y^{\sigma}\right]=\left[x^{-1} z_{1}, y^{-1} z_{2}\right]=\left[x^{-1}, y^{-1}\right]=[x, y]$ (for some $z_{1}, z_{2} \in \Phi(R)$ ), that is, $\sigma$ centralizes $R^{\prime}=\Phi(R)$.

(5) $Q P$ centralizes $\Phi(R)$.

By (3) and (4), $[\Phi(R), Q P] \leq\left(C_{G}(\sigma)\right)^{\prime}$. Now $C_{G}(\sigma)$ admits $D_{8} /\langle\sigma\rangle$ f.p.f. so Bauman's Theorem tells us that $[\Phi(R), Q P] \leq F_{1}\left(C_{G}(\sigma)\right) \cdot C_{S}(\sigma)$ is non-trivial, for otherwise $\sigma$ would invert $S$ and therefore commute with the automorphisms of $S$ induced by $R Q P$, against (4). So $C_{S}(\sigma)$ is non-trivial and lies, with $[\Phi(R), Q P]$, in $F_{l}\left(C_{G}(\sigma)\right)$. Since $r \neq s$ we deduce that $[\Phi(R), Q P]$ centralizes $C_{S}(\sigma)$. But $[\Phi(R), Q P] \triangleleft R Q P D_{8}$ so the irreducibility of $S$ implies that $[\Phi(R), Q P]$ centralizes $S$, contrary to (2) unless $[\Phi(R), Q P]=1$.

At this point it is convenient to pass to a finite splitting field $F$ for $R Q P D_{8}$ and its subgroups, of characteristic $s$; and to a faithful irreducible $R Q P D_{8}$-submodule $S^{*}$ say, of $S \otimes_{G F(s)} F$. The condition that $D_{8}$ act f.p.f. on $S$, namely that $\sum_{\alpha \in D_{8}} \alpha$ be the zero transformation, 
remains invariant under these manoeuvres, so $D_{8}$ acts f.p.f. on $S^{*}$.

(6) $R$ is not elementary abelian.

Let $W$ be an RQP-homogeneous component of $S^{*}$ and $D_{1}$ the stabilizer of $W$ in $D_{8}$. So $W$ is an irreducible $R Q P D_{1}$-module and $W=W_{1}+\ldots+W_{n}$ where the $W_{i}$ are isomorphic irreducible RQP-modules. The number of isomorphism types of irreducible $R$-submodules of $W_{i}$ is prime to 2 , so $D_{1}$ stabilizes an $R$-homogeneous component $V$ say, of $W \cdot S^{*}$ is a faithful $R$-module, irreducible for $R Q P D_{8}$, therefore $R$ acts f.p.f. on $S^{*}$ and so $R$ acts non-trivially on $V$.

If $D_{1}=1$, then for any non-trivial element $w \in W, \sum_{\alpha \in D_{8}} w_{\alpha}$ is a non-trivial fixed-point of $D_{8}$ in $S^{*}$, contrary to our initial assumption.

Now suppose $\sigma \notin D_{1}$, so we may assume without loss of generality that $D_{1}=(\eta)$. Then a non-trivial fixed-point $w \in W$ of $\eta$ would yield a non-trivial fixed-point $w+w \tau+w \sigma+w \sigma \tau \in S^{*}$ of $D_{8}$, so $\eta$ must act f.p.f. on $W$, therefore $\eta$ inverts $W$ and hence centralizes $R Q P / \operatorname{ker}(R Q P$ on $W)$. Therefore $n$ centralizes $Q P / \operatorname{ker}(Q P$ on $W), \quad n^{\tau}=n \sigma$ centralizes $Q P / \operatorname{ker}(Q P$ on $W \tau), \eta^{\sigma}=n$ centralizes $Q P / \operatorname{ker}(Q P$ on $W \sigma)$ and $n^{\sigma \tau}=n \sigma$ centralizes $Q P / \operatorname{ker}(Q P$ on $W \sigma \tau)$. However, by (3), $\sigma$ centralizes $Q P$ so $n$ itself centralizes these quotients. Therefore $n$ centralizes $Q P$ (because $i^{*}=W+W \tau+W \sigma+W \sigma \tau$ is a faithful $Q P-$ module) and so $Q P$ admits $D_{8} /\langle\sigma, n\rangle$ f.p.f. which is impossible since QP is not abelian.

We have thus shown that $\sigma \in D_{1}$. Suppose $R$ is elementary abelian. $V$ is a homogeneous $R$-module, non-trivial for $R$, so $1 \neq R / \operatorname{ker}(R$ on $V)$ is cyclic and represented by scalar transformations. Therefore $\sigma$ centralizes this quotient (whether $\sigma$ is trivial on $V$ or not) against (4). 
(7) T centralizes" $\Phi(R)$ and $R$ is extraspecial.

Our aim is to show that $\eta$ and $\eta \tau$ act f.p.f. on $\Phi(R)$, from which (7) follows readily. By $(4), C_{\Phi(R)}(\eta)=C_{\Phi(R)}(\langle\eta, \sigma\rangle) \triangleleft R$, so by (5), $C_{\Phi(R)}((\eta, \sigma))$ \& $R Q P D_{8}$. If the four-group $\langle\eta, \sigma\rangle$ acts f.p.f. on $S^{*}$ then it acts f.p.f. on an $R Q P\langle\eta, \sigma\rangle$-homogeneous component, $U$ say, of $S^{*}$. Now we may apply Theorem 4.1 of Shult [7] to deduce that some element, $\omega$ say, of $\langle\eta, \sigma\rangle$ centralizes $R Q P / \operatorname{ker}(R Q P$ on $U)$. If $\omega=\sigma$ then $\sigma=\sigma^{\tau}$ also centralizes $R Q P / \operatorname{ker}(R Q P$ on $U \tau)$, so $\sigma$ centralizes $R Q P$ (because $S^{*}=U+U T$ is a faithful $R Q P$-module) against (4). If $\omega=n$ or $n \sigma$ then an argument like that used in the proof of $(6)$ yields a contradiction. Thus $C_{S^{*}}(\langle\eta, \sigma\rangle)$ is non-trivial.

Now $C_{S^{*} R Q P}(\langle\eta, \sigma\rangle)$ admits $D_{8} /\langle\eta, \sigma\rangle$ f.p.f. so it is abelian. Therefore $C_{\Phi(R)}(\langle n, \sigma\rangle)$ centralizes $C_{S^{*}}(\langle n, \sigma\rangle)$. In view of the normality $C_{\Phi(R)}(\langle\eta, \sigma\rangle) \triangleleft R Q P D_{8}$ and the irreducibility of $S^{*}$, we must have $C_{\Phi(R)}(\langle\eta, \sigma\rangle)=1$, so $\eta$ acts f.p.f. on $\Phi(R)$. Thus $\eta$ inverts each element of $\Phi(R)$. But by the same argument so doe.; $n \tau$, therefore $\tau$ centralizes $\Phi(R)$ as we require. This means that $D_{8}$ inverts $\Phi(R)$ so, in view of (4) and (5), it follows that each subgroup of $\Phi(R)$ is normal in $R Q P D_{8}$. Therefore each element of $\Phi(R)$ acts f.p.f. on $S^{*}$, that is, $\Phi(R)$ acts regularly on $S^{*}$. (4) and (6) establish that $R$ is a non-abelian special group, so by 5.3 .14 of [4], $\Phi(R)$ is cyclic of order $r$.

(8) $S^{*}$ is the sum of 2 homogeneous components, $S_{1}^{*}$ and $S_{2}^{*}$ say, under $\Phi(R)$ and $\tau$ acts f.p.f. on $S^{*}$.

Since, by $(4),(5)$ and $(7), R Q P(\tau)$ centralizes $\Phi(R), S^{*}$ is either a homogeneous $\Phi(R)$-module or is the sum of 2 homogeneous components. In the first case $\Phi(R)$ acts as scalar transformations of $S^{*}$, so the transformations representing $\Phi(R)$ conmute with those representing $D_{8}$, that is, $D_{8}$ centralizes $\Phi(R)$, contradicting the f.p.f. action of $D_{8}$ on $G$. Therefore $S^{*}=S_{1}^{*}+S_{2}^{*}$ say, the 
$\Phi(R)$-homogeneous components $S_{1}^{*}$ and $S_{2}^{*}$ stabilized by and irreducible under $R Q P(\tau)$, and interchanged by $\eta$. If $v$ is a non-trivial element of $S_{1}^{*}$ centralized by $\tau$ then $v+v n$ is a non-trivial element of $S^{*}$ centralized by $D_{8}$ again contrary to assumption. Similarly $\tau$ acts f.p.f. on $S_{2}^{*}$ and so (8) is established.

Our final contradiction follows from

(9) $R$ has order $3^{3}$.

Let $S_{0}^{*}$ be an irreducible $R(\tau)$-submodule of $S_{1}^{*}$. Since $S^{*}$ is an irreducible $R Q P D_{8}$-module, $\Phi(R)$ acts f.p.f. on $S^{*}$, so $S_{0}^{*}$ is a faithful $R$-module and hence, by (4), also faithful for $R\langle\tau\rangle$. Because $S_{0}^{*}$ is homogeneous for $\Phi(R)$ and $R$ is extraspecial, it follows that $S_{0}^{*}$ is a homogeneous $R$-module. (The $r-1$ faithful irreducible representations of $R$ are characterized by the actions of $\Phi(R)$.) Since an irreducible projective representation of a cyclic group is 1-dimensional, the analogue of Theorem 51.7 of [3] in characteristic $s$ shows that $S_{0}^{*}$ is actually an irreducible $R$-module. By (4), $T$ acts regularly on the non-trivial elements of $R / \Phi(R)$ and by $(8), \tau$ acts f.p.f. on $S_{0}^{*}$. These last three facts enable us to use the Hall-Higman type argument of Shult [7] in his proof of Theorem 3.1 to deduce that $R$ has order $3^{3}$.

Now by (9) the chief factor $R / \Phi(R)$ of $G D_{8}$ has order $3^{2}$ and must therefore be centralized by $Q$, against (le). This final contradiction establishes the theorem.

\section{References}

[1] S.F. Bauman, "The Klein group as an automorphism group without fixed point", Pacific J. Math. 18 (1966), 9-13.

[2] T.R. Berger, "Nilpotent fixed point free automorphism groups of solvable groups", (to appear). 
[3] Charles W. Curtis, Irving Reiner, Representation theory of finite groups and associative algebras (Pure and Applied Mathematics, XI. Interscience [John Wiley \& Sons], New York, London, 1962).

[4] Daniel Gorenstein, Finite groups (Harper and Row, New York, Evanston, London, 1968).

[5] Fletcher Gross, "Fixed-point-free operator groups of order 8 ", Pacific J. Math. 28 (1969), 357-361.

[6] Fletcher Gross; "Elementary abelian operator groups", Bull. Austral. Math. Soc. 7 (1972), 91-100.

[7] Ernest E. Shult, "On groups admitting fixed point free abelian operator groups", IZlinois J. Math. 9 (1965), 701-720.

School of Mathematics and Physics,

University of East Anglia,

Norwich,

England. 